\title{
Fluorescence confocal microscopy for pathologists
}

\author{
Moira Ragazzi ${ }^{1,5}$, Simonetta Piana ${ }^{1,5}$, Caterina Longo ${ }^{2}$, Fabio Castagnetti ${ }^{3}$, Monica Foroni ${ }^{3}$, \\ Guglielmo Ferrari $^{3}$, Giorgio Gardini ${ }^{1}$ and Giovanni Pellacani ${ }^{4}$ \\ ${ }^{1}$ Pathology Unit, IRCSS Santa Maria Nuova Hospital, Reggio Emilia, Italy; ${ }^{2}$ Skin Cancer Unit, IRCSS Santa \\ Maria Nuova Hospital, Reggio Emilia, Italy; ${ }^{3}$ Breast Surgery Unit, IRCSS Santa Maria Nuova Hospital, Reggio \\ Emilia, Italy and ${ }^{4}$ Department of Dermatology, University of Modena and Reggio Emilia, Reggio Emilia, Italy
}

\begin{abstract}
Confocal microscopy is a non-invasive method of optical imaging that may provide microscopic images of untreated tissue that correspond almost perfectly to hematoxylin- and eosin-stained slides. Nowadays, following two confocal imaging systems are available: (1) reflectance confocal microscopy, based on the natural differences in refractive indices of subcellular structures within the tissues; (2) fluorescence confocal microscopy, based on the use of fluorochromes, such as acridine orange, to increase the contrast epithelium-stroma. In clinical practice to date, confocal microscopy has been used with the goal of obviating the need for excision biopsies, thereby reducing the need for pathological examination. The aim of our study was to test fluorescence confocal microscopy on different types of surgical specimens, specifically breast, lymph node, thyroid, and colon. The confocal images were correlated to the corresponding histological sections in order to provide a morphologic parallel and to highlight current limitations and possible applications of this technology for surgical pathology practice. As a result, neoplastic tissues were easily distinguishable from normal structures and reactive processes such as fibrosis; the use of fluorescence enhanced contrast and image quality in confocal microscopy without compromising final histologic evaluation. Finally, the fluorescence confocal microscopy images of the adipose tissue were as accurate as those of conventional histology and were devoid of the frozen-section-related artefacts that can compromise intraoperative evaluation. Despite some limitations mainly related to black/white images, which require training in imaging interpretation, this study confirms that fluorescence confocal microscopy may represent an alternative to frozen sections in the assessment of margin status in selected settings or when the conservation of the specimen is crucial. This is the first study to employ fluorescent confocal microscopy on surgical specimens other than the skin and to evaluate the diagnostic capability of this technology from pathologists' viewpoint.
\end{abstract}

Modern Pathology (2014) 27, 460-471; doi:10.1038/modpathol.2013.158; published online 13 September 2013

Keywords: breast; colorectum; confocal microscopy; fluorescence confocal microscopy; lymph node; surgical pathology; thyroid

Confocal microscopy is an optical method that can provide high-resolution images of fresh, non-fixed, thick pieces of tissue, which are optically sliced, instead of using a microtome blade. This is possible because the specimen is scanned with the help of a point source of light, specifically a laser, and thanks to a pinhole, which rejects out-of-focus light, a thin

Correspondence: Dr M Ragazzi, MD, Pathology Unit, IRCSS Santa Maria Nuova Hospital, Viale Risorgimento, 80, Reggio Emilia 42123, Italy.

E-mail: moira.ragazzi@asmn.re.it

${ }^{5}$ These authors contributed equally to this work.

Received 27 April 2013; revised 7 July 2013; accepted 8 July 2013; published online 13 September 2013 section of the tissue is obtained, analyzed using software, and displayed on a monitor. Confocal microscopy has been used mainly in research laboratories; however, its application in clinical settings has been also reported. ${ }^{1-26}$

The following two confocal imaging systems are currently available: (1) reflectance confocal microscopy, based on the natural differences in refractive indices of subcellular structures within a given tissue; (2) fluorescence confocal microscopy, based on the use of fluorochromes, such as acridine orange, to increase the cell-to-stroma contrast. Reflectance confocal microscopy has been mainly used for in vivo accessible tissue examination, finding its natural application first in ophthalmology, where it 
is currently used to inspect the retina and the cornea, ${ }^{1-3}$ in dermatology, ${ }^{4}$ where it is applied in the in vivo diagnosis of neoplastic and inflammatory skin diseases, ${ }^{5-7}$ and in the exploration of body cavities reachable through probe-based confocal laser endomicroscopes. ${ }^{8-16}$ Compared with the wider use of the in vivo confocal microscopy, ex vivo tissue assessment has been performed mainly in skin oncology to examine excision

Table 1 Type of tissues, number of acquisitions per specimens, and pathological diagnosis of analyzed samples

\begin{tabular}{lcl}
\hline $\begin{array}{l}\text { Tissues } \\
(\mathrm{n}=35)\end{array}$ & $\begin{array}{c}\text { Mean of acquired } \\
\text { images per sample }\end{array}$ & $\begin{array}{l}\text { Pathologic diagnosis } \\
\text { (number of cases) }\end{array}$ \\
\hline Breast (12) & 2.0 & $\begin{array}{l}\text { Non-neoplastic (4) } \\
\text { IDC GI (1) }\end{array}$ \\
& & IDC GII (2) \\
& IDC GIII (1) \\
& DCIS (1) \\
& ILC (2) \\
& & LCIS (1) \\
& & Goiter (3) \\
Hyperplastic nodule (3) & PTC-CV (2) \\
Thyroid (8) & 2.0 & Invasive adenocarcinoma (7) \\
& & Non metastatic (4) \\
Colon (7) & 1.6 & Metastatic (4) \\
\hline
\end{tabular}

Abbreviations: DCIS, ductal carcinoma in situ; G, histological grade; IDC, invasive ductal carcinoma; ILC, invasive lobular carcinoma; LCIS, lobular carcinoma in situ; PTC-CV, papillary thyroid carcinoma-classical variant.

${ }^{\mathrm{a}}$ Metastases were from IDC (three axillary lymph nodes) and from PTC (one cervical lymph node). margins in Mohs surgery; ${ }^{17}$ only few studies have focused on different tissues. ${ }^{18-26}$

In surgical oncology, the possibility to obtain a reliable histopathologic diagnosis in a short time represents a relevant clinical advantage for patient management. Nowadays histopathologic diagnosis is routinely rendered through intraoperative frozen tissue examination when a rapid diagnosis is required. The introduction of confocal microscopy offers the possibility to analyze fresh tissues at histopathologic resolution while avoiding tissue processing.

The aim of our study was to explore fluorescence confocal microscopy on different types of surgical specimens, specifically breast, thyroid, colon, and lymph nodes. The confocal images were correlated to the corresponding histological sections in order to provide a morphologic parallel and to highlight current limitations and possible applications of this technology for diagnostic surgical pathology practice.

\section{Materials and methods}

\section{Case Selection}

A total of 35 surgical specimens from 30 patients (4 male and 26 female patients) were analyzed at the Pathology Unit of the Arcispedale Santa Maria Nuova-IRCCS, Reggio Emilia, Italy. Altogether 12 breast lumpectomies, 8 thyroids, 7 colorectal resections, and 8 lymph nodes (6 axillary and 2 cervical lymph nodes) were collected. In order not to delay routine patient management when intraoperative diagnosis was required, two contiguous fresh
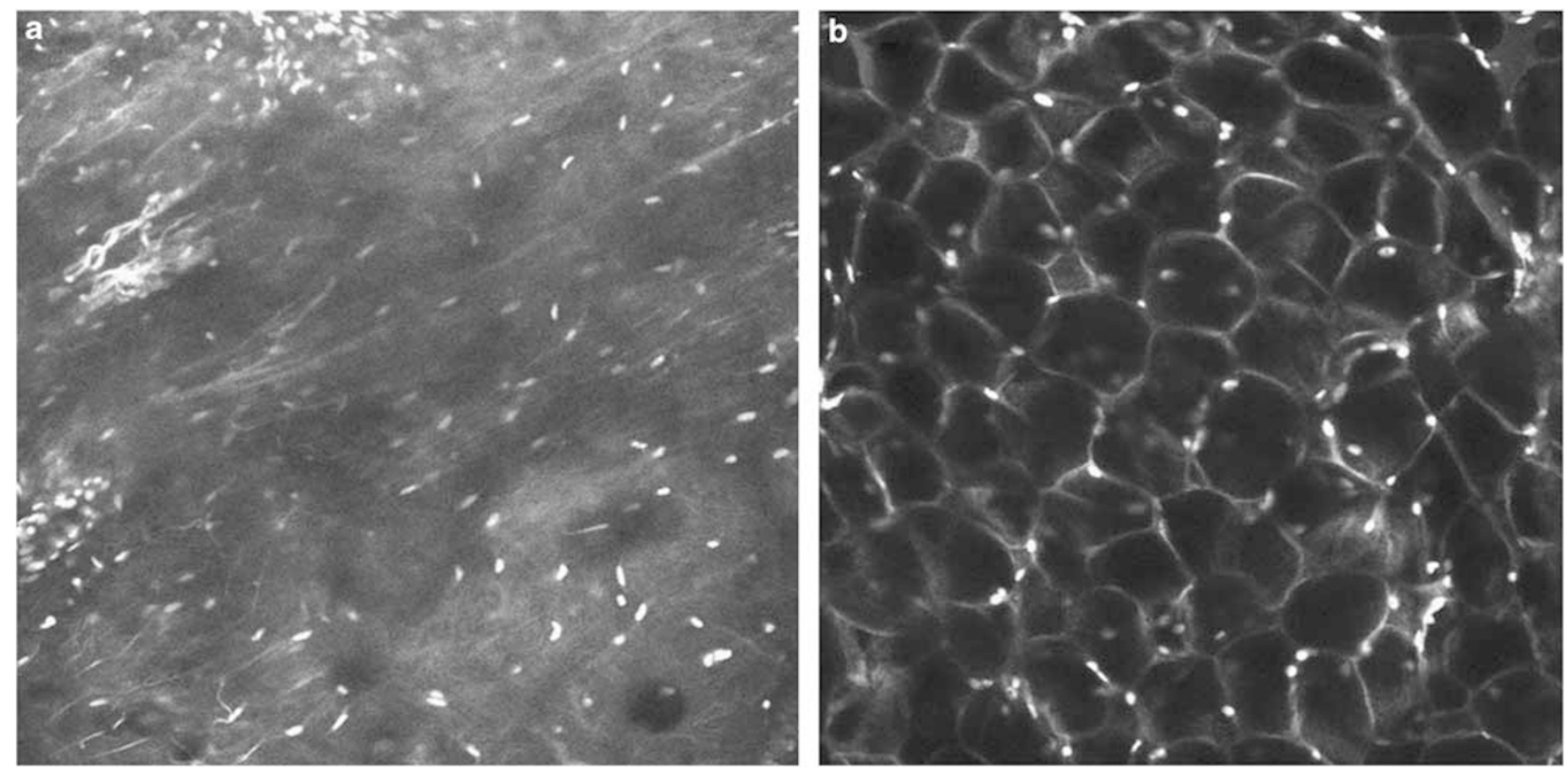

Figure 1 Stromal tissues on fluorescence confocal microscopy. (a) Fibrous tissue appears as hypofluorescent bundles including oval bright spots, which correspond to fibroblast nuclei. (b) Adipose tissue appears as black spaces surrounded by a thin network of fluorescent lines. 

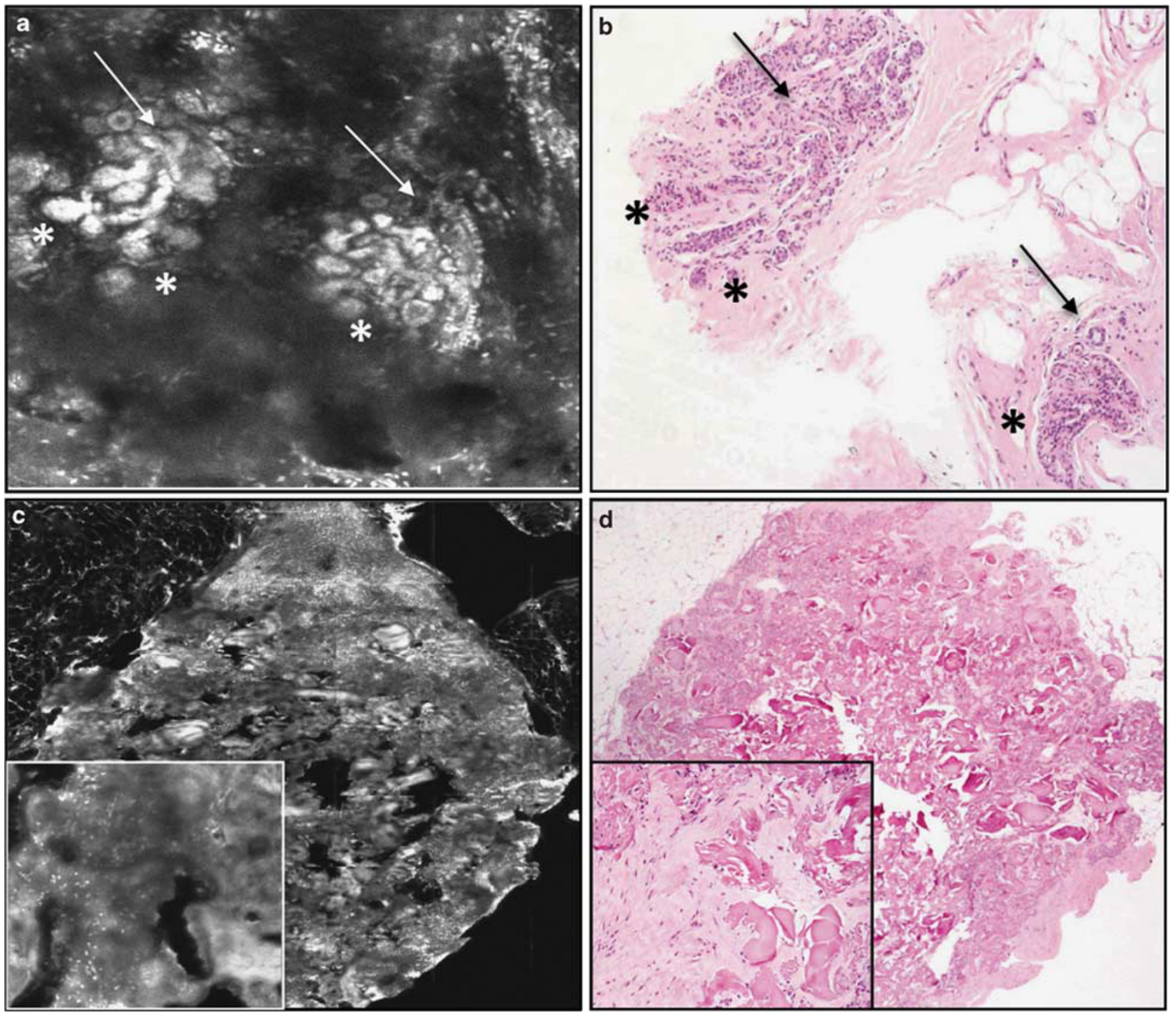

Figure 2 Non-neoplastic breast. (a and b) On fluorescence confocal microscopy, normal lobules (asterisk) are seen as fluorescent roundish spots at the end of elongated ductal structures (arrows) on a black-gray background, which corresponds to fibro-fatty breast tissue (a). The same features can be seen on the H\&E-stained slide (b). (c and d) On fluorescence confocal microscopy, H\&E mammotome sponge, made up of inert material, appears as a roundish mass with a distinct border (c) and a low degree of brightness when compared with scattered fluorescent spindle fibroblast nuclei (inset). The morphologic overlap with the H\&E findings is impressive (d).

samples were taken for each specimen-one for the frozen histology and one for the confocal analysis.

In breast and colorectal resections, the slice was selected from the tumor and/or the nearest surgical margin; samples from thyroids were obtained from a representative area of the lesion, whereas lymph nodes were entirely analyzed in one or more slides according to their size.

In three cases of breast lumpectomy and three cases of lymph node resection, the same section was used first for fluorescence confocal microscopy and then for conventional frozen examination.

The study was conducted in accordance with the precepts of the Helsinki Declaration (http://www. wma.net/en/30publications/10policies/b3/) and all data were handled anonymously.

\section{Fluorescence Confocal Microscopy}

The confocal microscope used in this study was the VivaScope 2500 (Caliber I.D., Rochester, New York, USA). This instrument is characterized by an inverted microscope setup connected to the computer software VivaScan. The images were scanned in two dimensions along $x$ and $y$ axes by using a diode laser illumination at a wavelength of $488 \mathrm{~nm}$ (bluefluorescence wavelength) with a $\times 30,0.9$ numerical aperture water immersion objective lens. The horizontal optical resolution was $<2 \mu \mathrm{m}$ at the center of field of view, and the vertical (axial) optical resolution was $<5 \mu \mathrm{m}$ at the center of field of view. The maximum depth of imaging was up to $250 \mu \mathrm{m}$, depending on tissue type. 

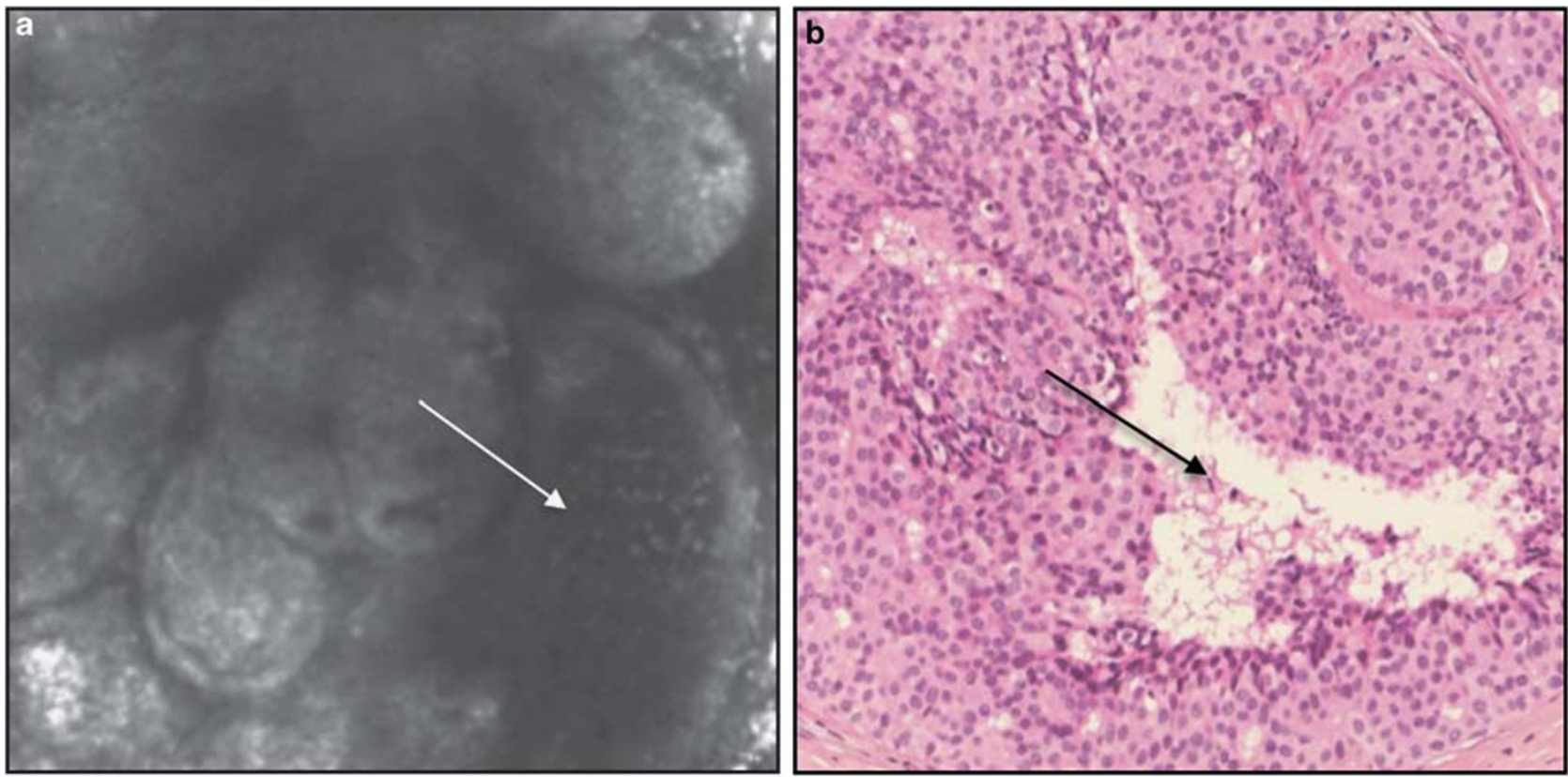

Figure 3 Ductal carcinoma in situ of the breast. (a and b) The bright aggregates on fluorescence confocal microscopy (a) morphologically correspond to the ducts enlarged by monomorphous neoplastic cells on H\&E (b). The arrow indicates an irregular lumen with cellular debris.

A representative slice of the lesional area, maximum size $1.2 \mathrm{~cm}$ (height) $\times 1.2 \mathrm{~cm}($ width $) \times 0.3 \mathrm{~cm}$ (thickness), was dipped for $20 \mathrm{~s}$ in acridine orange $0.6 \mathrm{mM}$, as this was demonstrated to be the optimum concentration to improve the quality of digital staining. ${ }^{27}$ The excess dye was removed using a tissue paper. The slice was then placed between two silicon-sealed glass slides. These slides were mounted on a specific support of the confocal microscope. A sequence of high-resolution images $(0.75 \mathrm{~mm} \times 0.75 \mathrm{~mm})$ was automatically acquired, scanning an area up to $12 \times 12 \mathrm{~mm}$ (mosaic). ${ }^{28}$ The acquired mosaic, which corresponds to a standard low-power $\times 2$ view of optical microscopes, was evaluated by 'zooming' in (visualizing a squared area of $0.75 \times 0.75 \mathrm{~mm}^{2}$ ) and out (visualizing a squared area of $1.5 \times 1.5 \mathrm{~mm}^{2}$ ) at a resolution of $1000 \times 1000$ pixels. This 'zooming' mimics the manual changing of objective magnification during the usual histologic slide-reading process on conventional light microscopy. Acridine orange, which binds nucleic acids and in particular DNA, is a well-proven nuclear stain for confocal microscopy. The fluorescent signal from the cellular nuclei is converted to grayscale information by using a computer software. As a result, the image brightness or intensity was proportional to the content of cellular nuclei in the tissue: when the nuclei were packed together, as in a lymph node or in a tumoral tissue, the fluorescence was high and the images were bright. In the absence of a fluorescent signal-that is, when the tissue was hypocellular as in fibrous or adipose tissues-the background was darker because the signal intensity was less.

\section{Histopathology Examination}

Frozen-Section Analysis. For six specimens (three lymph nodes and three breast resections), a snapfrozen section was obtained after fluorescence confocal microscopy and before formalin fixation to verify whether acridine orange would affect the frozen-section evaluation.

Formalin-Fixed and Paraffin-Embedded Analyses. The tissues previously analyzed using fluorescence confocal microscopy were then formalin-fixed and paraffin-embedded; the same space orientation used for confocal analysis was maintained, and a $3-\mu \mathrm{m}-$ thick slice was stained with H\&E. The diagnosis was rendered according to specific WHO criteria. ${ }^{29-31}$

\section{Results}

Details on the cases, number of acquisitions per specimen, and pathological diagnosis are summarized in Table 1. For each sample, image acquisition was repeated one to three times (mean 1.84), in order to obtain an interpretable mosaic. When repeated images were collected from the same specimen, a variable photo bleaching occurred in four cases. To obviate this issue, the specimen was dipped again in acridine orange. Notably, the number of acquisitions to obtain a suitable fluorescence confocal microscopy image was about the same for the different types of tissues analyzed. It took up to $9 \mathrm{~min}$ to analyze a maximum-sized specimen $(1.2 \mathrm{~cm} \times 1.2$ $\mathrm{cm})$ and to obtain a single mosaic. As confocal and 
histological sections maintained the same tissue orientation, the fields compared were almost exactly the same, despite the minimum loss of material during microtome slicing.

All confocal images were reviewed by two pathologists, and morphological features were compared with those of the corresponding pathological sections.

The main types of stromal tissues present in all specimens analyzed were fibrous and adipose tissues. On fluorescence confocal microscopy they were both clearly recognizable. Fibrous tissue was composed of hypofluorescent bundles including oval bright spots, which corresponded to fibroblast nuclei (Figure 1a). Adipose tissue showed up as black non-fluorescent spaces surrounded by a thin network of bright lines (Figure 1b). This dark background represented an optimum contrast for the easy identification of cell-enriched tissues, such as neoplastic proliferations.
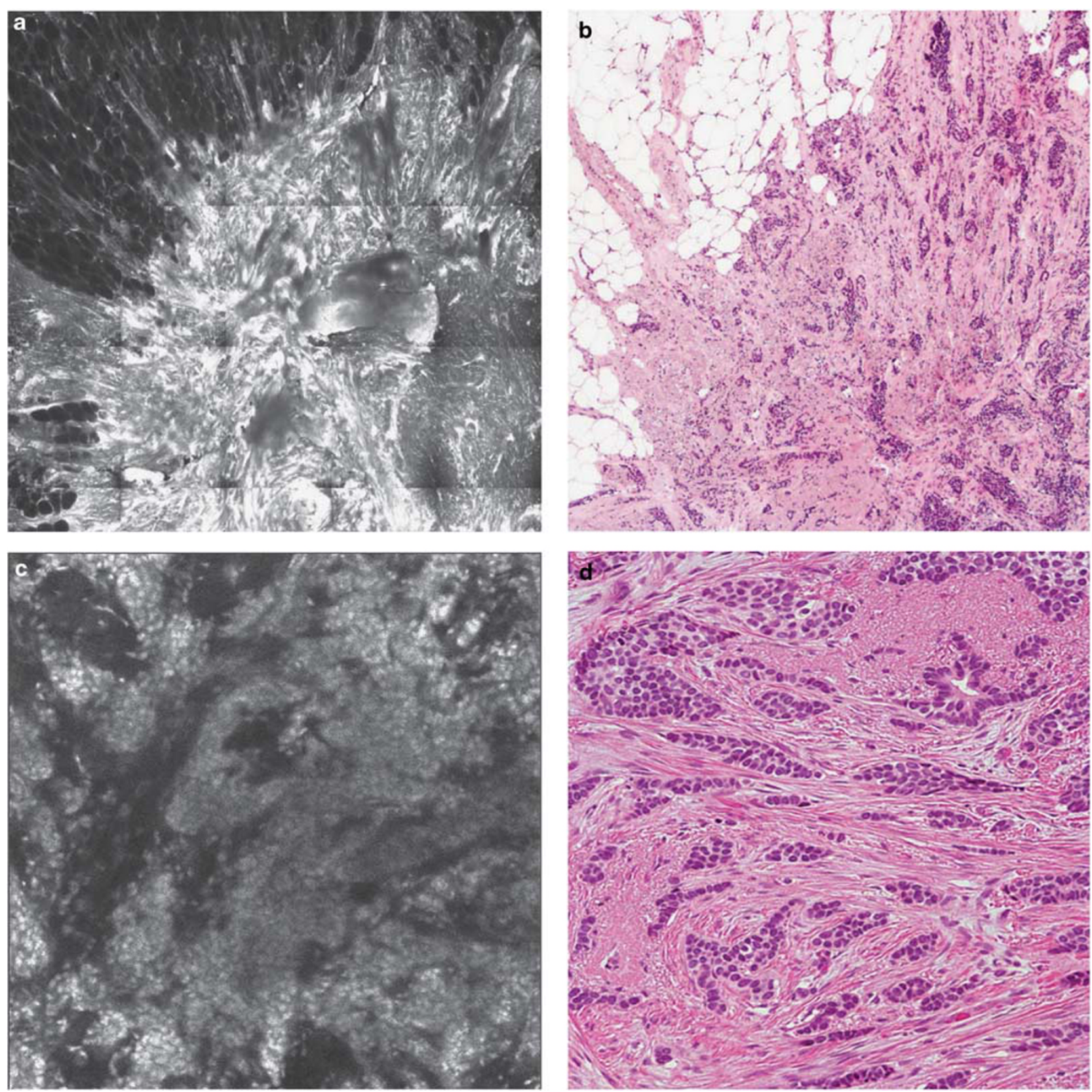

Figure 4 Invasive breast carcinoma. (a and b) Invasive duct carcinoma at low magnification: the elongated bright bands on a dark background (a) correspond to the neoplastic ducts infiltrating the adipose tissue (b). (c and d) Invasive duct carcinoma at high magnification: bright, irregular ribbons are separated by gray stalks (c). On conventional histology, the invasive ducts are evident (d) on a desmoplastic stroma. (e and f) Invasive lobular carcinoma at high magnification: bright cords of cells infiltrate a dark background (e) with the same pattern of growth as that seen in H\&E staining (f). 


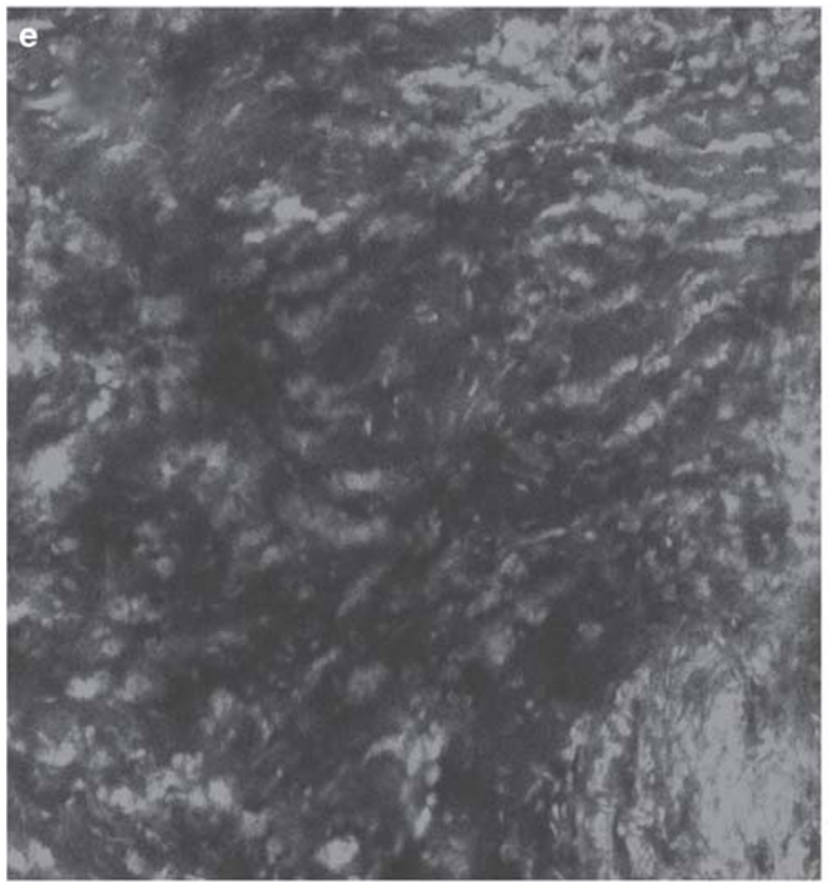

Figure 4 Continued.

As acridine orange is a nuclear stain, nuclear size and shape could be clearly evaluated, whereas cytoplasmic details were not so well defined.

\section{Breast}

Concerning breast specimens, four cases consisted of non-neoplastic parenchyma (three cases were surgical margins without neoplasia and one case was an inflammatory reaction around a mammotome sponge). On fluorescence confocal microscopy, normal breast lobules appeared as roundish structures composed of bright spots at the end of elongated ductal structures (Figure 2a). They clearly stood out against the background of the adipose tissue, which was hypofluorescent and dark. These same features corresponded perfectly to those detected in the H\&E-stained slice (Figure 2b). Likewise, the foreign material of a mammotome sponge was easily recognized as a greyish nodule including only scattered fluorescent elongated nuclei, corresponding to fibroblasts on H\&E staining (Figures 2c and d).

Considering neoplastic breast specimens, two out of twelve were in situ carcinomas (one ductal carcinoma in situ and one lobular carcinoma in situ), four were invasive ductal carcinomas, and two invasive lobular carcinomas upon histological examination.

On fluorescence confocal microscopy, in situ carcinomas showed well-defined, bright, roundish structures that corresponded to the ducts and lobules distended by highly fluorescent neoplastic cells (Figure 3a). In ductal carcinoma in situ, a

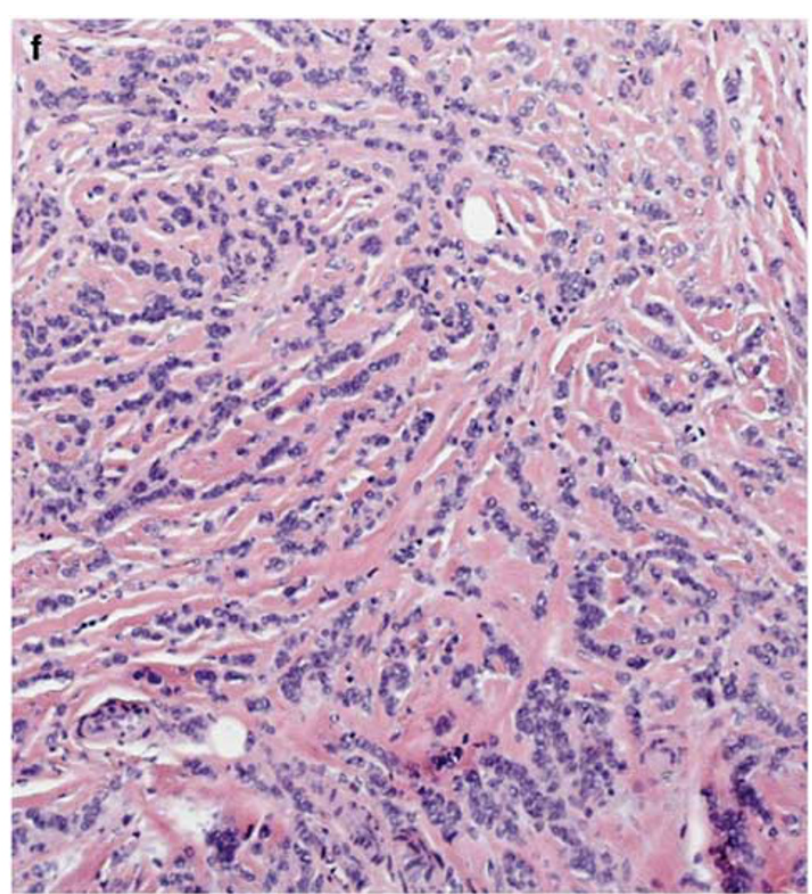

hypofluorescent lumen could be seen, which was morphologically superimposable to the irregular lumen filled with debris observed in the H\&E section (Figure 3b).

In invasive carcinomas, the neoplastic areas were easily detectable on fluorescence confocal microscopy as a bright mass with irregular borders, composed of many fluorescent neoplastic nuclei (Figure 4a). The proliferating cells stood out from the surrounding hypofluorescent fibroadipose tissue, exactly as the infiltrating nests and cords within the adipose tissue do on pathological examination (Figure 4b).

At high magnification, tubular and glandular structures of invasive ductal carcinomas, and infiltrating cords of the invasive lobular carcinomas, were recognizable in confocal images as well as in $\mathrm{H} \& \mathrm{E}$ slides (Figures 4c-f). In high-grade invasive carcinomas, nuclear pleomorphism was distinguishable as irregularly shaped bright dots, larger than those observed in well-differentiated carcinomas.

\section{Thyroid}

Pathological examination revealed three cases of colloid goiter, three cases of hyperplastic nodules, and two cases of papillary carcinoma-classical variant.

On fluorescence confocal microscopy, non-neoplastic parenchyma was composed of dark spaces containing rare light dots (follicles filled with colloid material and cellular debris) encircled by a single layer of bright spots that corresponded to the nuclei of thyrocytes (Figures 5a and b). In papillary carcinomas, papillary structures were distinguishable as 

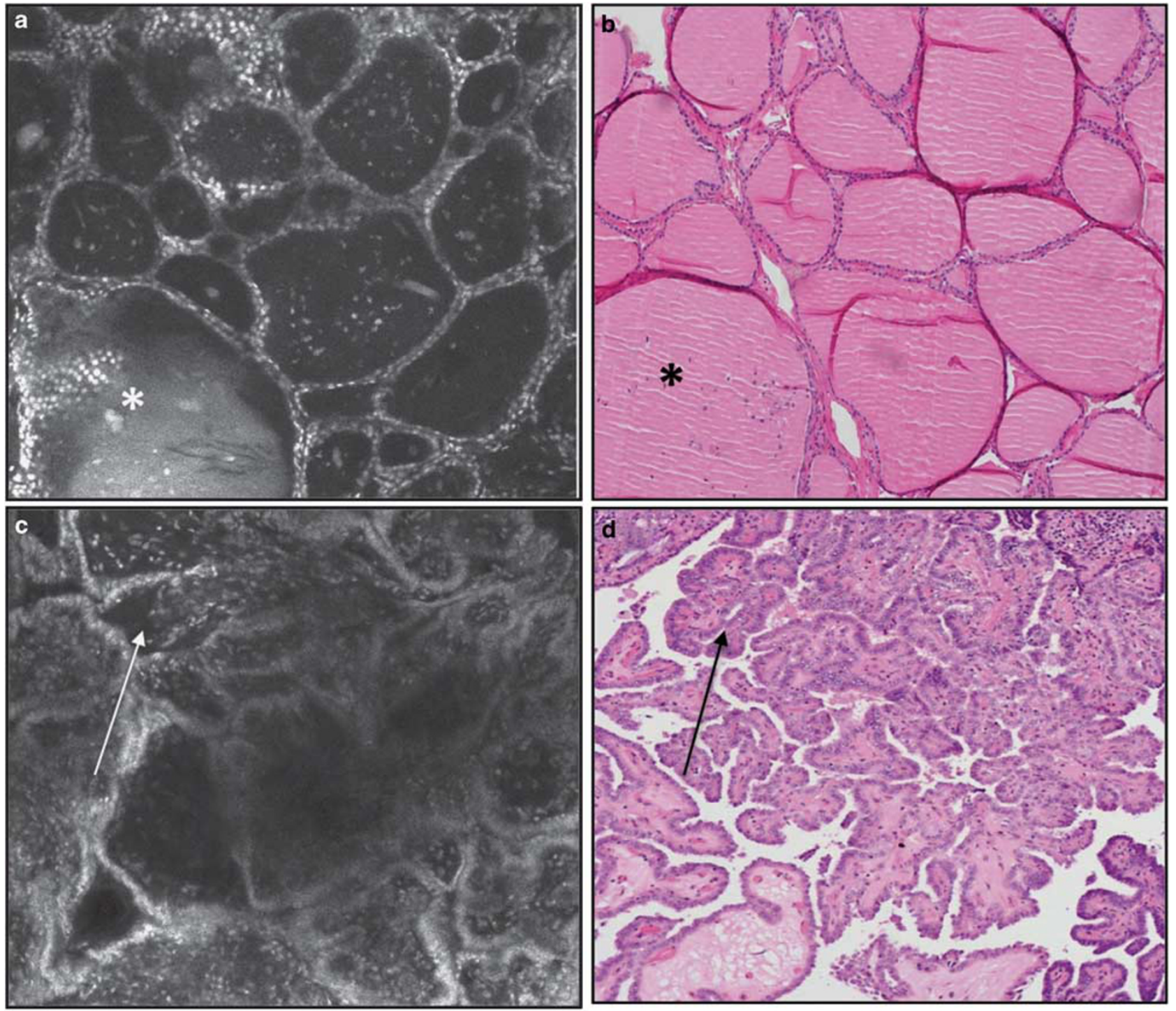

Figure 5 Normal and neoplastic thyroid. (a and b) On fluorescence confocal microscopy, non-neoplastic parenchyma appears as dark spaces (asterisk) encircled by a single layer of bright spots (a). These spaces correspond to normal follicles surrounded by thyrocytes and filled with colloid material and cellular debris (b). The morphological correspondence is remarkable. (c and d) In papillary carcinomas, papillary structures are identifiable as projections made up of scanty bright spots (arrow) and surrounded by a thicker, irregular bright line (c). This corresponds to the fibroblasts of the fibrovascular stalk and pseudostratified neoplastic thyrocytes on H\&E evaluation (d).

digitiform projections made up of scanty bright spots (nuclei of fibroblasts that make up fibrovascular stalk) and surrounded by a thicker, irregular bright line (pseudostratified neoplastic nuclei) (Figures 5c and d).

\section{Colorectum}

All the cases under evaluation were colorectal resections for invasive adenocarcinoma. On fluorescence confocal microscopy, normal colonic glands appeared as small, regular, roundish spaces surrounded by a single layer of bright spots (Figures 6a and b) and were easily distinguishable from adjacent neoplastic areas. The latter presented as irregular, bright, randomly distributed glandular structures with the typical 'back-to-back' feature. These findings were confirmed in the H\&E picture (Figures 6c and d).

\section{Lymph Nodes}

On pathological examination, four out of eight lymph nodes were reactive. On fluorescence confocal microscopy, normal lymph node parenchyma was composed of fluorescence spots, corresponding to small lymphocytes, and by irregular stalks with a lesser degree of brightness, corresponding to fibrous septa and sinus histiocytosis (Figures 7a and b).

Breast invasive ductal carcinoma metastases were observed in three axillary lymph nodes. In metastatic 



Figure 6 Normal and neoplastic colorectum. (a and b) On fluorescence confocal microscopy of normal colonic mucosa (a), small round spaces surrounded by a single layer of bright spots (arrow) correspond exactly to glands evenly spaced, with central regular lumen, in H\&E (b). (c and d) In colon adenocarcinoma, randomly distributed irregular bright glandular structures with the typical 'back-to-back' feature can be recognized on fluorescence confocal microscopy (c) and are confirmed by H\&E image (d).

lymph nodes, fluorescent spots were bigger and irregularly shaped with a more cohesive architecture, as expected for carcinomatous cells (Figures 7c and d). In one cervical lymph node, hyperfluorescent papillary structures were evident in dark cystic spaces (Figure 7e) and corresponded to a cystic lymph node metastasis from thyroid papillary carcinoma (Figure 7f).

\section{Discussion}

Histopathologic diagnosis basically relies on the identification of specific morphological criteria that has been validated among the scientific community. Thus, the definition of morphological criteria as seen upon fluorescence confocal microscopy is mandatory to obtain a consistent diagnosis.

When using a confocal microscope, pathologists have to be trained to correlate the H\&E-stained purple nuclei to acridine orange-stained nuclei, and the eosin-stained pink cytoplasms or connective structures to shades of gray, as they appear in digital images rendered with computer software.

The possibility to enhance nuclei visibility makes this tool suitable for rapid tumor detection. In the present study, we sought to evaluate the feasibility of this promising tool in tissues other than the skin.

In our series, the majority of the analyzed cases were represented by breast parenchyma. Normal and neoplastic breast tissues have been previously 
evaluated using reflectance confocal microscopy on core biopsies. ${ }^{18,19}$ We confirmed that neoplastic invasive carcinomas can be easily distinguished from normal ducts and that neoplastic areas are distinguishable from reactive processes such as fibrosis, making also possible to discriminate mammotome sponge.
Unlike Schiffauer et al, ${ }^{19}$ who used reflectancemode device, we found that breast adipose tissue did not cause optical aberrations with fluorescence confocal microscopy. In fact, the fluorescence confocal microscopy images of the adipose tissue were as accurate as those in conventional histology and did not present frozen-section-related artefacts
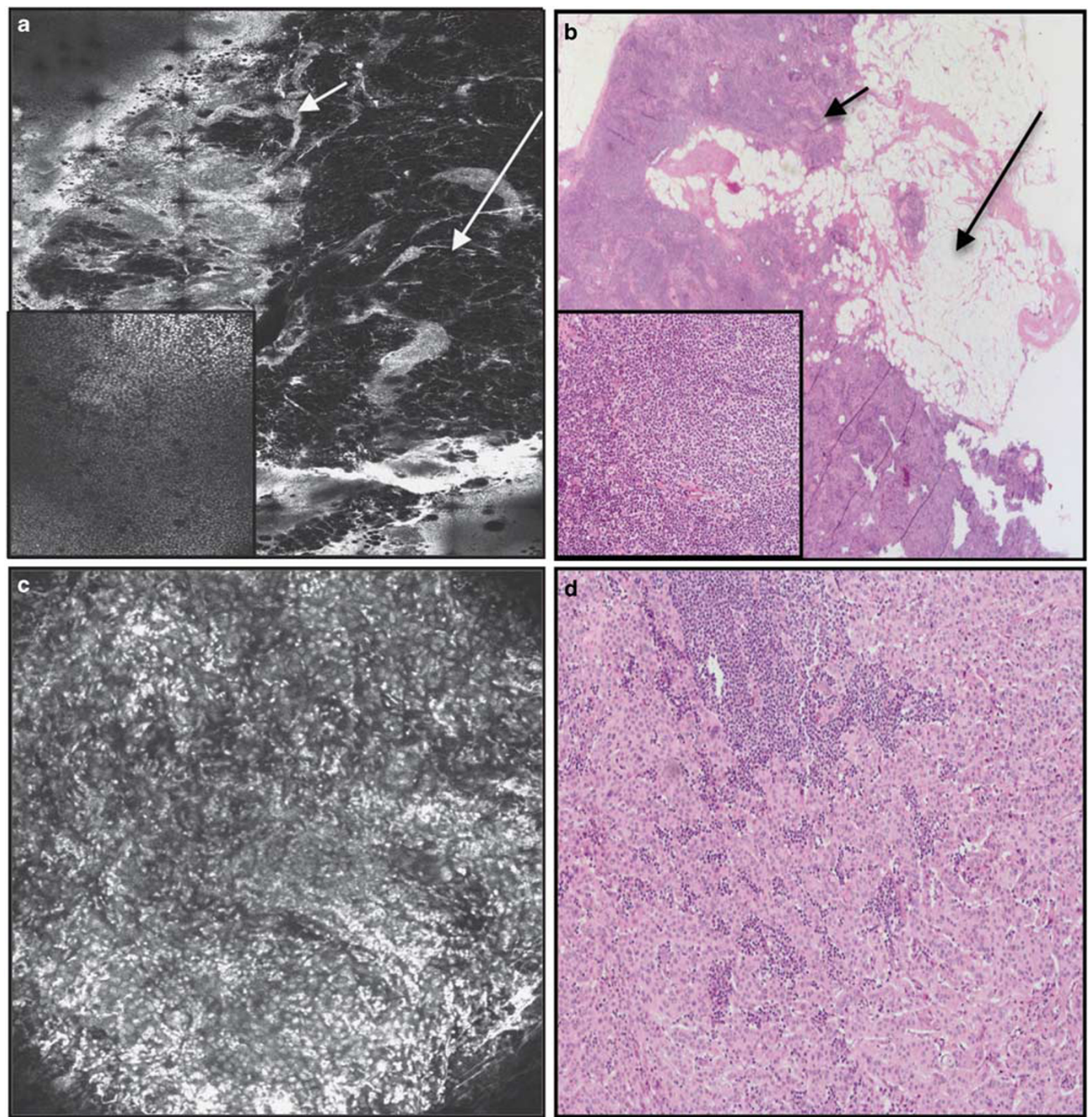

Figure 7 Normal and metastatic lymph node parenchyma. (a and b) On fluorescence confocal microscopy, normal lymph node parenchyma appears as sheets of bright spots, corresponding to small lymphocytes (inset), intermingled with irregular stalks with a lesser degree of brightness, corresponding to fibrous septa and sinus histiocytosis (small arrow). The perinodal fat tissue (big arrow) is black on fluorescence confocal microscopy. (c and d) Invasive ductal carcinomas' metastatic lymph nodes at high magnification: bright spots are bigger and irregular and show a more cohesive pattern (c). In H\&E section, the lymph node parenchyma is almost completely replaced by carcinomatous cells (d). (e and f) Lymph node metastases from thyroid carcinoma are papillary structures made up of scanty bright spots floating in a dark background. 

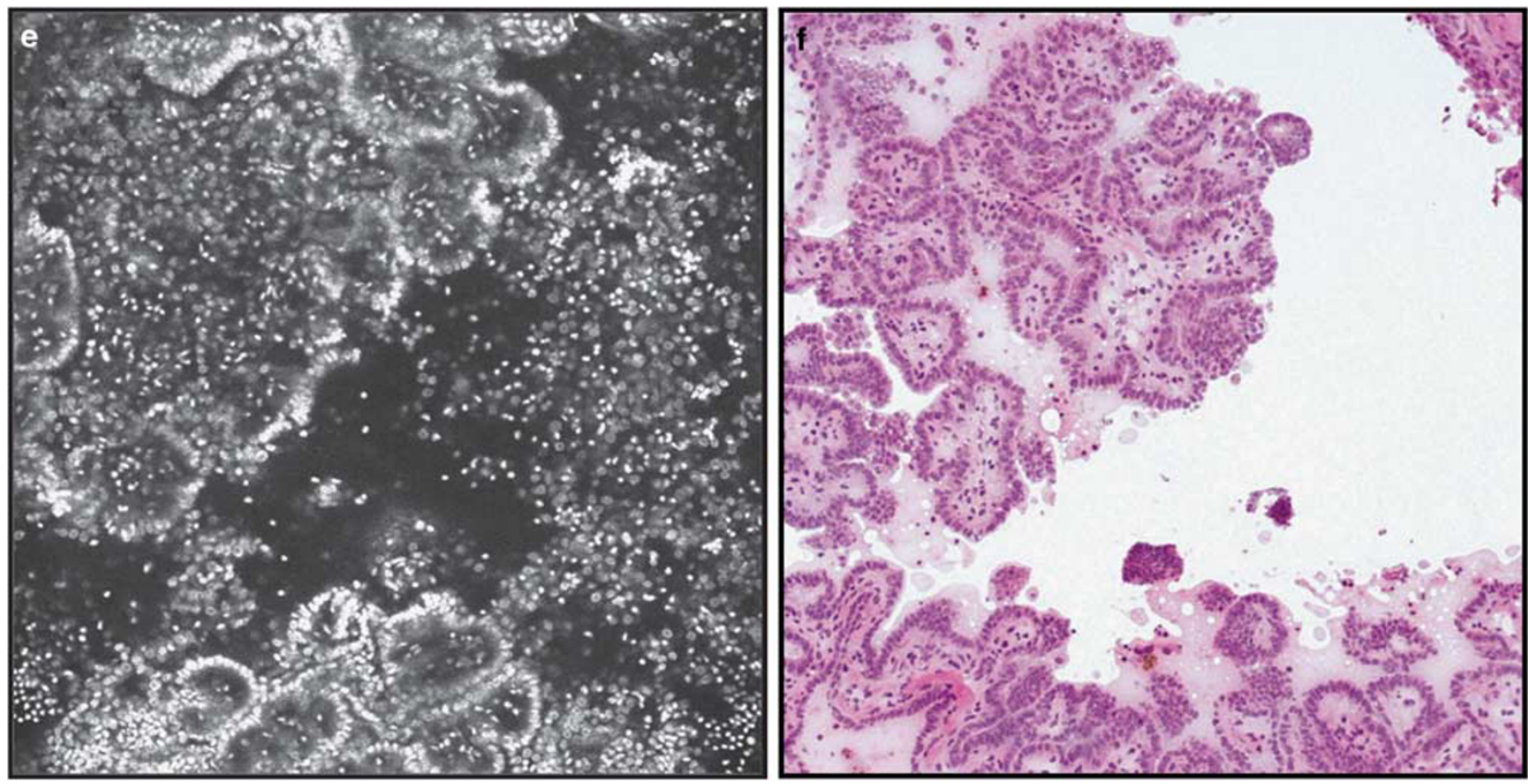

Figure 7 Continued.

that may compromise the intraoperative evaluation of surgical margins in conventional breast conserving surgery.

Lymph node represented the most challenging specimen on fluorescence confocal microscopy image analysis. The main issue derived from the high nuclear density, which generated intense fluorescence that may masquerade small metastatic deposits. Metastases were recognizable, basing on the detection of cellular, nuclear pleomorphism, and/or characteristic neoplastic architecture, such as for tubular and glandular structures in breast cancer. Therefore, fluorescence confocal microscopy seemed not yet appropriate to diagnose metastatic invasive lobular carcinomas because of discohesive pattern of growth and monotonous cellularity, not distinguishable from normal lymphocytes. The development of immunohistochemical techniques (for example, anti-cytokeratin immunostaining) applied to fluorescence confocal microscopy could improve lymph node analysis.

A pilot study assessed the use of reflectance confocal microscopy to describe normal thyroid tissue, with results that encouraged broader application. $^{22}$ Our study included two cases of papillary carcinoma as well, which is the most frequent thyroid malignancy. On fluorescence confocal microscopy images, the papillary architecture appeared as projections, made of scanty fluorescence spots, corresponding to the fibroblasts, outlined by a fluorescent thick border, corresponding to overlapped thyrocytes nuclei, that were clearly distinguishable from normal follicles. As the confocal technique cannot evaluate nuclear detail, it is not possible to identify follicular variants of papillary carcinoma, a limit that should also be taken into consideration in frozen-section analysis.

As fluorescence confocal microscopy imaging permits easy identification of the border between tumor and normal mucosa in colorectal resection, the evaluation of the margins is also feasible in gastrointestinal surgical pathology. Thus, a possible application of fluorescence confocal microscopy could be for intraoperative examination, when quick preliminary histologic evaluations are required by the surgeon.

Overall, fluorescence confocal microscopy offers some advantages over frozen-section analysis: a shorter turnaround time; the preservation of tissue integrity for routine histological examination; and easy detection of adipose tissue, which is usually difficult to achieve in routine frozen sections. Moreover, we found that the acridine orange -staining process did not alter tissues in any way and the diagnoses were not adversely affected. Based on our experience, fluorescence confocal microscopy seemed to be a promising alternative to frozen sections in the evaluation of margin status in breast and colorectal cancer types, in small breast tumors or thyroid lesions $(<1 \mathrm{~cm})$, where the preservation of the tissue integrity is of outmost importance and frozen sections are not indicated, and in adipose lymph nodes, where frozen-section analysis is often unfeasible.

However, some limitations need to be considered. Primarily pathologists need to learn to read and interpret black/white images and to evaluate a tissue with less cytological details than $\mathrm{H} \& \mathrm{E}$-stained sections. To overcome this obstacle, a dedicated software capable of digitally stain fluorescence confocal microscopy images has been recently 
developed and tested on basal cell carcinoma, rendering tissue pictures almost perfectly overlapping with conventional histopathology. ${ }^{32}$ Last but not least, the grade of invasiveness of a neoplasia often cannot be evaluated on fluorescence confocal microscopy, as the window of the microscope $(12 \mathrm{~mm} \times 12 \mathrm{~mm})$ is smaller than the size of the specimens.

New microscopic technologies are being developed rapidly for the use in many fields of medicine. In surgical pathology, confocal microscopy can be viewed as an adjunct to conventional histopathology to hasten the evaluation of surgical margins and to avoid performing frozen-section examination in small neoplastic lesions. Explorative and prospective studies on many different tissues are necessary to determine whether fluorescence confocal microscopy is appropriate and clinically advantageous, looking forward to further technical improvements.

\section{Acknowledgements}

We thank Ms Jacqueline Costa (IRCSS Santa Maria Nuova Hospital, Reggio Emilia, Italy) for the English-language revision of the article.

\section{Disclosure/conflict of interest}

The authors declare no conflict of interest.

\section{References}

1 Webb RH, Hughes GW, Delori FC. Confocal scanning laser ophthalmoscope. Appl Opt 1987;26:1492-1499.

2 Sunness JS, Schuchard RA, Shen N, et al. Landmarkdriven fundus perimetry using the scanning laser ophthalmoscope. Invest Ophthalmol Vis Sci 1995;36:1863-1874.

3 Cavanagh HD, Petroll WM, Alizadeh H, et al. Clinical and diagnostic use of in vivo confocal microscopy in patients with corneal disease. Ophthalmology 1993;100:1444-1454.

4 Rajadhyaksha M, Grossman M, Esterowitz D, Webb RH, Anderson RR. In vivo confocal scanning laser microscopy of human skin: melanin provides strong contrast. J Invest Dermatol 1995;104:946-952.

5 Pellacani G, Guitera P, Longo C, et al. The impact of in vivo reflectance confocal microscopy for the diagnostic accuracy of melanoma and equivocal melanocytic lesions. J Invest Dermatol 2007;127:2759-2765.

6 Guitera P, Menzies SW, Longo C, et al. In vivo confocal microscopy for diagnosis of melanoma and basal cell carcinoma using a two-step method: analysis of 710 consecutive clinically equivocal cases. J Invest Dermatol 2012;132:2386-2394.

7 Longo C, Zalaudek I, Argenziano G, Pellacani G. New directions in dermatopathology: in vivo confocal microscopy in clinical practice. Dermatol Clin 2012;30:799-814; viii.
8 Thiberville L, Moreno-Swirc S, Vercauteren T, et al. In vivo imaging of the bronchial wall microstructure using fibered confocal fluorescence microscopy. Am J Respir Crit Care Med 2007;175:22-31.

9 Thiberville L, Salaun M, Lachkar S, et al. Human in vivo fluorescence microimaging of the alveolar ducts and sacs during bronchoscopy. Eur Respir J 2009;33:974-985.

10 Kiesslich R, Hoffman A, Goetz M, et al. In vivo diagnosis of collagenous colitis by confocal endomicroscopy. Gut 2006;55:591-592.

11 Kiesslich R, Goetz M, Burg J, et al. Diagnosing Helicobacter pylori in vivo by confocal laser endoscopy. Gastroenterology 2005;128:2119-2123.

12 Kakeji Y, Yamaguchi S, Yoshida D, et al. Development and assessment of morphologic criteria for diagnosing gastric cancer using confocal endomicroscopy: an ex vivo and in vivo study. Endoscopy 2006;38:886-890.

13 Kitabatake S, Niwa Y, Miyahara R, et al. Confocal endomicroscopy for the diagnosis of gastric cancer in vivo. Endoscopy 2006;38:1110-1114.

14 Kiesslich R, Burg J, Vieth M, et al. Confocal laser endoscopy for diagnosing intraepithelial neoplasias and colorectal cancer in vivo. Gastroenterology 2004;127:706-713.

$15 \mathrm{Wu}$ K, Liu JJ, Adams W, et al. Dynamic real-time microscopy of the urinary tract using confocal laser endomicroscopy. Urology 2011;78:225-231.

16 Carlson K, Pavlova I, Collier T, et al. Confocal microscopy: imaging cervical precancerous lesions. Gynecol Oncol 2005;99:S84-S88.

17 Karen JK, Gareau DS, Dusza SW, et al. Detection of basal cell carcinomas in Mohs excisions with fluorescence confocal mosaicing microscopy. Br J Dermatol 2009;160:1242-1250.

18 Tilli MT, Cabrera MC, Parrish AR, et al. Real-time imaging and characterization of human breast tissue by reflectance confocal microscopy. J Biomed Opt 2007;12:051901.

19 Schiffhauer LM, Boger JN, Bonfiglio TA, et al. Confocal microscopy of unfixed breast needle core biopsies: a comparison to fixed and stained sections. BMC Cancer 2009;9:265.

20 Bickford LR, Agollah G, Drezek R, Yu TK. Silica-gold nanoshells as potential intraoperative molecular probes for HER2-overexpression in ex vivo breast tissue using near-infrared reflectance confocal microscopy. Breast Cancer Res Treat 2010;120:547-555.

21 White WM, Tearney GJ, Pilch BZ, et al. A novel, noninvasive imaging technique for intraoperative assessment of parathyroid glands: confocal reflectance microscopy. Surgery 2000;128:1088-1100; discussion 100-101.

22 White WM, Baldassano M, Rajadhyaksha $\mathrm{M}$, et al. Confocal reflectance imaging of head and neck surgical specimens. A comparison with histologic analysis. Arch Otolaryngol Head Neck Surg 2004;130:923-928.

23 Campo-Ruiz V, Ochoa ER, Lauwers GY, et al. Evaluation of hepatic histology by near-infrared confocal microscopy: a pilot study. Hum Pathol 2002;33:975-982.

24 Keck T, Campo-Ruiz V, Warshaw AL, et al. Evaluation of morphology and microcirculation of the pancreas by ex vivo and in vivo reflectance confocal microscopy. Pancreatology 2001;1:48-57.

25 Anuthama K, Sherlin HJ, Anuja N, et al. Characterization of different tissue changes in normal, betel chewers, potentially malignant lesions, conditions 
and oral squamous cell carcinoma using reflectance confocal microscopy: correlation with routine histopathology. Oral Oncol 2010;46:232-248.

26 El Hallani S, Poh CF, Macaulay CE, et al. Ex vivo confocal imaging with contrast agents for the detection of oral potentially malignant lesions. Oral Oncol 2013;49:582-590.

27 Bini J, Spain J, Nehal K, et al. Confocal mosaicing microscopy of human skin ex vivo: spectral analysis for digital staining to simulate histology-like appearance. J Biomed Opt 2011;16:076008.

28 Gareau DS, Li Y, Huang B, et al. Confocal mosaicing microscopy in Mohs skin excisions: feasibility of rapid surgical pathology. J Biomed Opt 2008;13:054001.
29 Lakhani SR, Ellis IO, Schnitt SJ, et al. Classification of Tumours of the Breast, 44th edn. World Health Organization: Lyon, France, 2012.

30 DeLellis RA, Lloyd RV, Heitz PU, et al. World Health Organization Classification of Tumours. Pathology and Genetics of Endocrine Organs. IARC: Lyon, France, 2004.

31 Bosman FT, Carneiro F, Hruban RH, et al. Classification of Tumours of the Digestive System, 34th edn. World Health Organization: Lyon, France, 2010.

32 Gareau DS, Jeon H, Nehal KS, et al. Rapid screening of cancer margins in tissue with multimodal confocal microscopy. J Surg Res 2012;178:533-538. 